\title{
Observations in homeotropic anchoring of the $+\pi$ disclinations in a hexagonal discotic liquid crystal $\left({ }^{*}\right)\left({ }^{* *}\right)$
}

\author{
P. Oswald \\ Laboratoire de Physique des Solides, Bât. 510, Université de Paris-Sud, \\ 91405 Orsay, France and S.E.S.I.- C.E.N., 92260 Fontenay-aux-Roses, France \\ (Reçu le 16 janvier 1981, accepté le 5 mars 1981)
}

\begin{abstract}
Résumé. - L'étude des défauts rectilignes et de leurs associations observés dans la phase hexagonale de l'hexapentoxytriphénylène a permis de montrer l'existence des lignes dièdre $S=\frac{1}{2}$ en développantes de cercle et de confirmer la symétrie $L 6$ de la phase discotique. L'énergie d'ancrage du discotique sur le verre non traité est évaluée $\left(\simeq 10^{-2} \mathrm{ergs} / \mathrm{cm}^{2}\right)$.
\end{abstract}

Abstract. - The study of the linear defects and their associations observed in the hexagonal phase of the hexa pentoxytriphenylene demonstrates the existence of $+\pi$ disclinations with molecular columns along evolutes of a circle and confirms the L 6 symmetry of the studied phase. The anchoring energy of the discotic on the glass is estimated $\left(\simeq 10^{-2} \mathrm{ergs} / \mathrm{cm}^{2}\right)$.

1. Introduction. - The observation between slide and coverslip of a hexagonal discotic liquid crystal reveals the existence of many linear defects.

First analysed by Billard et al. [1] they were recently given by Bouligand [2] a topological interpretation which we develop hereunder. We study successively a rectilinear surface defect and then associations of defects.

2. Material and methods. - The compound studied is the hexapentoxytriphenylene [1,3]. At normal pressure, the following transitions occur :

Solid crystal $\stackrel{69^{\circ} \mathrm{C}}{\rightleftarrows}$ hexagonal discotic phase $\stackrel{122^{\circ} \mathrm{C}}{\rightleftarrows}$ isotropic phase.

The samples are prepared between slide and coverslip. Their thickness is known precisely thanks to the utilization of calibrated mylar-foils. We have used an Orthoplan-Pol-Leitz polarizing microscope equipped with a rotating stage. The preparations were heated in a hot Mettler stage. For our observations the light is taken as parallel as possible by closing down the substage iris to the smallest size consistent with adequate illumination.

(*) Version anglaise d'une note présentée par Mr Y. Quéré à l'Académie des Sciences.

$\left({ }^{* *}\right)$ Work partially supported by DRET contract $79 / 352$.
3. Study of an isolated defect in homeotropic anchoring. - Our sample has a thickness of $75 \mu \mathrm{m}$. Figure 1 shows a linear defect the contrast of which is a maximum when :

- The light is polarized perpendicular to its axis. - We focus on the lower slide (Fig. 1a).

We observe the core of the defect. If we focus on the upper slide (Fig. 1b) a large zone of extinction is observed. The superposition of the two images ( $1 a$ and $1 b)$ shows that the defect axis is not in the centre of this dark zone but at one third of its width. If the polarization of the light is parallel to the defect axis, the defect is invisible whatever focussing might be.

\section{Interpretation of the results and topological model.} - These observations show that the optical axis is in a plane perpendicular to the defect axis. When the polarizer is parallel to the defect axis, only the ordinary ray propagates : being insensitive to the variations of direction of the director it is not deviated and the defect is invisible. When the polarizer is perpendicular to the defect axis, only the extraordinary ray is propagated. This latter one, which is sensitive to the variations of direction of the director, is strongly deviated near the core, twice as much on one side as on the other one.

All these results can be interpreted by considering that a $S=\frac{1}{2}$ line, along which the cylindrical rods are 


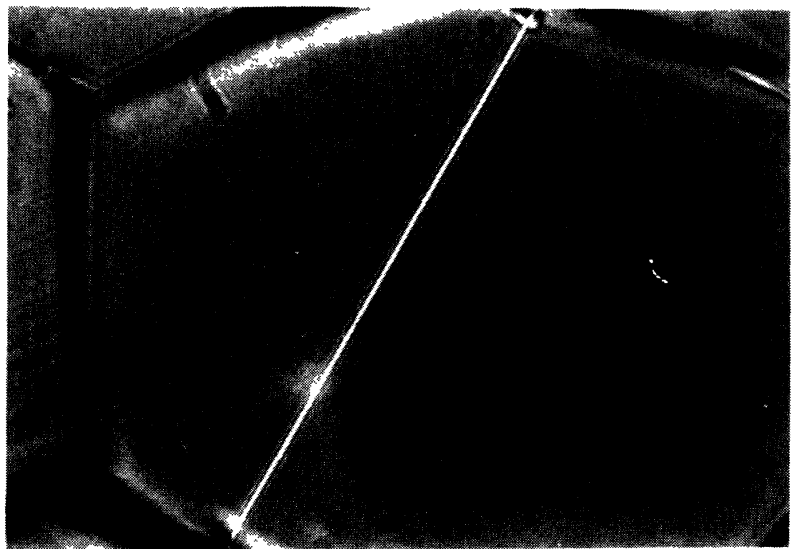

a)

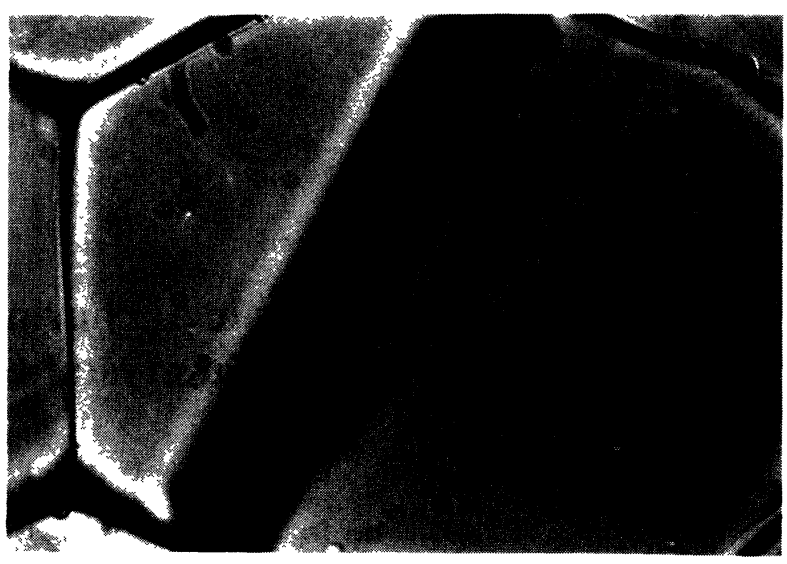

b)

Fig. 1. - Observation in parallel light of a linear surface defect. The polarization of the light is perpendicular to the defect axis. Only the extraordinary ray is propagated.

a) Focus on the lower slide; $b$ ) Focus on the upper slide.

curved in evolutes of a circle, lies on the bottom slide as indicated on figure 2 . This model is that of a defect of infinite extension which is cut by the two slides. It necessitates a low anchoring energy.

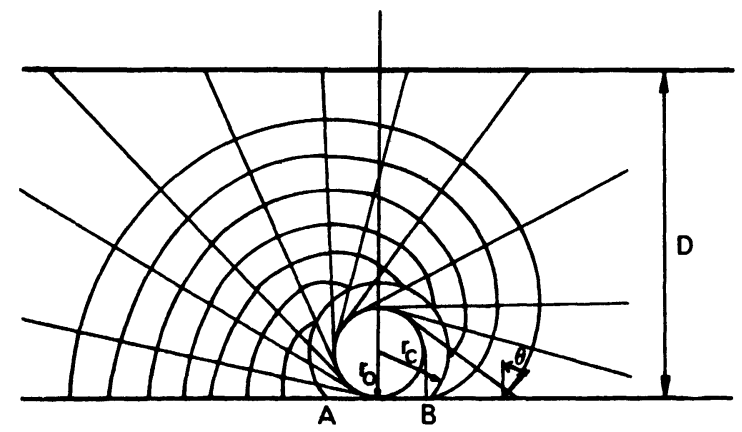

Fig. 2. - Line of strength $S=\frac{1}{2}$ lying on the slide (the cylindrical rods are curved in evolutes of a circle). In A the optical axis is perpendicular to the slide, in B it is parallel (which imposes $r_{0}=r_{\mathrm{c}} \frac{\sqrt{2}}{2}$ ).

A simplified model (Fig. 3), optically close to the one of figure 2 , consists in a concentric structure similar to that described by Grandjean [4]. He proved, by generalizing the Fermat principle, that the extraordinary rays follow the curve of polar equation :

$$
\rho=\frac{1}{a \cos \frac{\omega}{N}+b \sin \frac{\omega}{N}}
$$

where $N=n_{\mathrm{e}} / n_{0}$ is the ratio of the extraordinary index to the ordinary index. $a$ and $b$ are two unknown constants. We obtain a relation between $a$ and $b$ by writing that the tangential component of the wavevector $\mathbf{k}$ is continuous when entering the medium. We then prove easily that on the right (resp. left) the asymptote to the rays is the straight line of equation

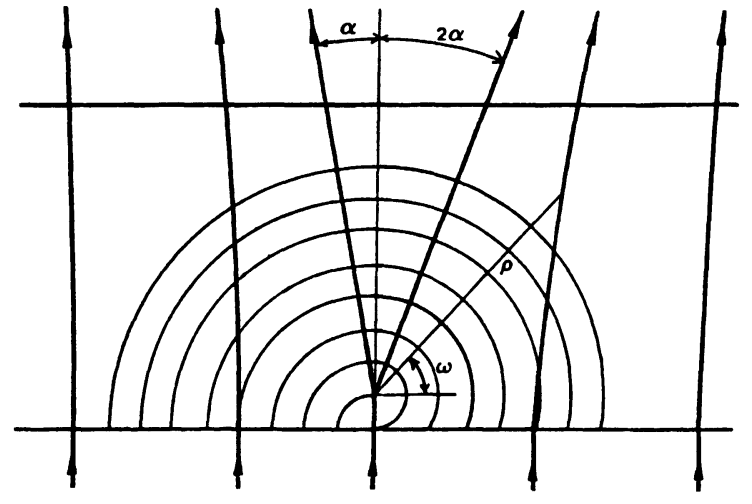

Fig. 3. - Simplified model used to describe the optical properties of a $S=\frac{1}{2}$ line lying on the slide. We have drawn the path of the extraordinary rays.

$\omega=\pi \frac{N}{2}\left(\right.$ resp. $\left.\omega=\frac{3 \pi}{2}-\pi N\right)$. This leads to a deviation of angle $\alpha=\frac{\pi}{2}(1-N)$ on the right of the core and to a deviation of angle $2 \alpha$ on the left. From figure 1 we measure $\alpha=8.3^{\circ}$ which leads to $N=0.907$. We have $n_{0} \simeq 1.5$, whence the birefringence $\Delta n=-0.14$. We have measured directly [7] the birefringence with a Michelson interferometer $\left({ }^{1}\right)$ :

$$
|\Delta n|_{\text {measured }}=0.143 \pm 0.003 \text {. }
$$

The agreement is very good.

5. Calculation of the total energy and first estimate of the anchoring energy. - We note $D$ the thickness of the sample. Let us suppose that the "core is filled with hexagonal liquid crystal, the molecular cylinders being

( $\left.{ }^{1}\right)$ Kindly lent by J. Billard. 
parallel to the core axis [7]. This assumption will be discussed in a future article. According to the model of figure 2 the curvature energy of the defect reads :

$$
E_{\text {curvature }}=\frac{\pi k_{3}}{4} \ln \frac{D^{2}}{r_{\mathrm{c}}^{2}-r_{0}^{2}}+C
$$

where $C$ is a constant. In this calculation we have supposed $D \gg r_{0}$. It is necessary to add to this energy a core energy and an anchoring energy of the molecular cylinders on the slides. Let us assume that

$$
r_{0}=r_{\mathrm{c}} \frac{\sqrt{2}}{2}
$$

(Fig. 2); then the core energy reads :

$$
E_{\text {core }}=\frac{3 \pi}{2} r_{\mathrm{c}} \gamma
$$

We prove elsewhere that $\gamma$ is of the order of $7 \times 10^{-3} \mathrm{ergs} / \mathrm{cm}^{2}$ [7]. Let $\theta$ be the angle between the molecular cylinders and the normal to one of the slides and let us use the classical expression $W=A \sin ^{2} \theta$ as the anchoring energy function. Then the total anchoring energy reads :

$$
E_{\text {anchoring }}=\pi A D-2.5 A r_{0}+\circ\left(A \frac{r_{0}^{2}}{D}\right) \text {. }
$$

According to (3) the total energy is :

$E_{\text {total }}=\frac{\pi k_{3}}{2} \ln \frac{D}{r_{\mathrm{c}}}+\left(\frac{3 \pi}{2} \gamma-1.8 \mathrm{~A}\right) r_{\mathrm{c}}+$ const.

By minimizing $E_{\text {total }}$ with respect to the variable $r_{\mathrm{c}}$ we obtain :

$$
r_{\mathrm{c}}=\frac{\pi k_{3} / 2}{\frac{3 \pi}{2} \gamma-1.8 \mathrm{~A}} .
$$

On figure $1 a$ we measure $r_{\mathrm{c}}=1 \mu \mathrm{m}$. From [7]

$$
\gamma \sim 7 \times 10^{-3} \mathrm{ergs} / \mathrm{cm}^{2} \text { and } k_{3} \sim 10^{-6} \text { dynes } .
$$

According to (7) one gets $A \sim 10^{-2} \mathrm{ergs} / \mathrm{cm}^{2}$. This value is very small.

6. Associations of defects. - In the case of the thick samples ( $75 \mu \mathrm{m}$ for instance) we observe many associations of $+\pi$ disclinations. On figure 4 we have the

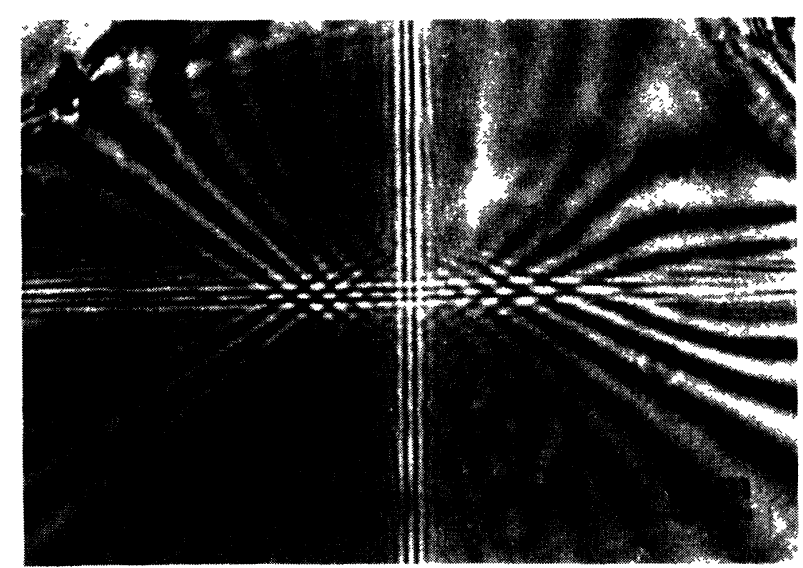

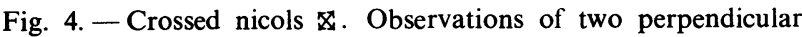
linear defects. Many interference fringes are visible.
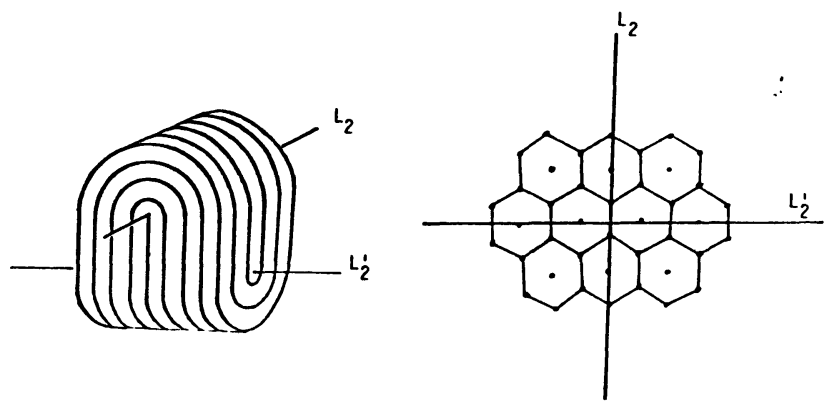

Fig. 5. - System of two $+\pi$ disclinations lying at right angle. Such a situation implies the presence of a $L 6$ axis (Bouligand [6]).

example of two $+\pi$ disclinations parallel to the slides, lying at right angle practically in the same plane. This situation is schematized on figure 5 . This association at right angle is relatively frequent. We have also observed associations at 30 and $60^{\circ}$. These results show obviously that the $\delta$ axis of a $+\pi$ disclination is not oriented at random but is on the contrary either a $L_{2}$ or $L_{2}^{\prime}$ axis of the hexagonal array (Fig. 5). These results are also a confirmation of the $\mathrm{L} 6$ symmetry of the discotic mesophase.

Acknowledgments. - The discotic product was kindly supplied by J.-C. Dubois from Thomson-C.S.F. Research Laboratories (Domaine de Corbeville, Orsay, France). Thanks are due to M. Kléman and Y. Quéré for their interest and useful discussions.

\section{References}

[1] Billard, J., Dubois, J.-C., Nguyen Huu Tinh et ZanN, A., Nouveau J. de Chimie 2, n" 5 (1978) 535.

[2] Bouligand, Y., J. Physique 41 (1980) 1307.

[3] Levelut, A. M., J. Physique Lett. 40 (1979) L-81.
[4] Grandjean, M. F., Bull. Soc. Fr. Mineral. 42 (1919) 42

[5] Kléman, M., J. Physique 41 (1980) 737.

[6] Bouligand, Y., J. Physique 41 (1980) 1297

[7] Oswald, P., Thèse de $3^{\mathrm{e}}$ cycle (1981). 